\title{
Microscopic Model for Photoinduced Magnetism in the Molecular Complex $\left[\mathrm{Mo}(\mathrm{IV})(\mathrm{CN})_{2}(\mathrm{CN}-\mathrm{CuL})_{6}\right]^{8+}$ Perchlorate
}

\author{
Rajamani Raghunathan ${ }^{\S}$, S. Ramasesha ${ }^{\S}$, Corine Mathonière ${ }^{\dagger}$, Valérie Marvaud M $^{*}$ \\ $\S$ Solid State and Structural Chemistry Unit, Indian Institute of Science, Bangalore 560 012, India \\ ${ }^{\dagger}$ Institut de Chimie de la Matière Condensée de Bordeaux CNRS UPR 9048 87, \\ Avenue du Docteur Schweitzer 33608 Pessac cedex (France) \\ ${ }^{\ddagger}$ Labaratoire de Chimie inorganique et Matériaux Moléculaires CNRS, \\ UMR 7071, Université Pierre et Marie Curie, 75252 Paris Cedex 05 (France)
}

\begin{abstract}
A theoretical model for understanding photomagnetism in the heptanuclear complex $\left[\mathrm{Mo}(\mathrm{IV})(\mathrm{CN})_{2}(\mathrm{CN}-\mathrm{CuL})_{6}\right]^{8+}$ perchlorate is developed. It is a many-body model involving the active orbitals on the transition metal ions. The model is exactly solved using a valence bond approach. The ground state solution of the model is highly degenerate and is spanned by five $\mathrm{S}=0$ states, nine $\mathrm{S}=1$ states, five $\mathrm{S}=2$ states and one $\mathrm{S}=3$ state. The orbital occupancies in all these states correspond to six $C u(I I)$ ions and one diamagnetic $M o(I V)$ ion. The optically excited charge-transfer (CT) state in each spin sector occur at nearly the same excitation energy of 2.993 $\mathrm{eV}$ for the physically reasonable parameter values. The degeneracy of the CT states is largest in the $\mathrm{S}=3$ sector and so is the transition dipole moment from the ground state to these excited states. Thus laser irradiation with light of this energy results in most intense absorption in the $\mathrm{S}=3$ sector. The life-time of the $\mathrm{S}=3$ excited states is also expected to be the largest as the number of states below that energy is very sparse in this spin sector when compared to other spin sectors. These twin features of our model explain the observed photomagnetism in the $\left[\mathrm{Mo}(\mathrm{IV})(\mathrm{CN})_{2}(\mathrm{CN}-\mathrm{CuL})_{6}\right]^{8+}$ complex.
\end{abstract}

(Received date : August 15, 2018)

\section{INTRODUCTION}

In the expanding field of molecular magnetism, photomagnetism has considerable import as it affords the possibility of magnetic switching with the aid of light. In the photomagnetic systems that we have studied here, the system before shining light is essentially paramagnetic, with negligible exchange interaction between the magnetic ions. Shining a burst of light of appropriate wavelength takes the system to a state in which there is a superexchange interaction between the paramagnetic ions. The photo-induced high-spin state is long lived at low temperatures, however, warming the system takes it back to the paramagnetic ground state [1]. There also exist extended systems in which the photoinduced exchange mechanism gives rise to magnetic ordering; this unusual photo-effect is called the Photo Induced Phase Transition (PIPT) 2].

The above phenomenon is quite distinct from the spin state transition observed in many inorganic complexes, wherein shining light leads to change in the ratio of the population of the low-spin (LS) state to that in the high-spin (HS) state. This phenomenon called the Light Induced Excited Spin State Trapping (LIESST), has been extensively studied in spin crossover complexes [3] . These systems lie very close to the spin crossover point in the Tanabe-Sugano diagram [4]. When the sys-

*E-mail: rajamani@sscu.iisc.ernet.in; ramasesh@sscu.iisc.ernet.in; mathon@icmcb-bordeaux.cnrs.fr; marvaud@ccr.jussieu.fr tem is excited by light, the non-radiative decay from the excited state occurs through intersystem crossings to the two nearly degenerate low energy states of different spin [5]. To recover the pure low-spin ground state, it would become necessary to warm the system and cool it to low temperatures. This phenomenon does not involve any magnetic exchange interactions between the transition metal ions.

Photomagnetism was experimentally first demonstrated in Fe-Co prussian blue complex $\mathrm{K}_{0.2} \mathrm{Co}_{1.4} \mathrm{Fe}(\mathrm{CN})_{6} \cdot 6.9 \mathrm{H}_{2} \mathrm{O}$. This system is ferrimagnetic with a $\mathrm{T}_{c}$ of $16 \mathrm{~K}$ [6]. Upon irradiation, the $\mathrm{T}_{c}$ increased to $19 \mathrm{~K}$. Besides, at fixed magnetic field and fixed temperature, the net magnetization increased upon irradiation. This increase in $\mathrm{T}_{c}$ and magnetization are attributed to electron transfer from the diamagnetic $F e(I I)$ to the diamagnetic $C o(I I I)$ brought about by irradiation resulting in $\mathrm{Fe}(I I I)$ ion with spin $1 / 2$ and $C o(I I)$ ion with spin $3 / 2$ [7]. These paramagnetic ions, besides contributing to an increase magnetization, also establish pathways for exchange which were blocked in the diamagnetic state, thereby increasing the $T_{c}$.

The system we have studied theoretically is the $\mathrm{Mo-}$ $\mathrm{Cu}$ molecular system. While photoinduced ferromagnetism has been observed in extended $\mathrm{Mo}-\mathrm{Cu}$ systems [2], we focus our attention in this paper on the molecular system $\left[\mathrm{Mo}(I V)(C N)_{2}(C N-C u(I I) L)_{6}\right]^{8+}$ with perchlorate as the counter ions [8]. In the ground state, each molecule consists of six noninteracting spins, derived from the six $C u(I I)$ ions; the $M o(I V)$ ion is diamagnetic. The magnetic behaviour of this molecule in the ground state corresponds to that of a system with 


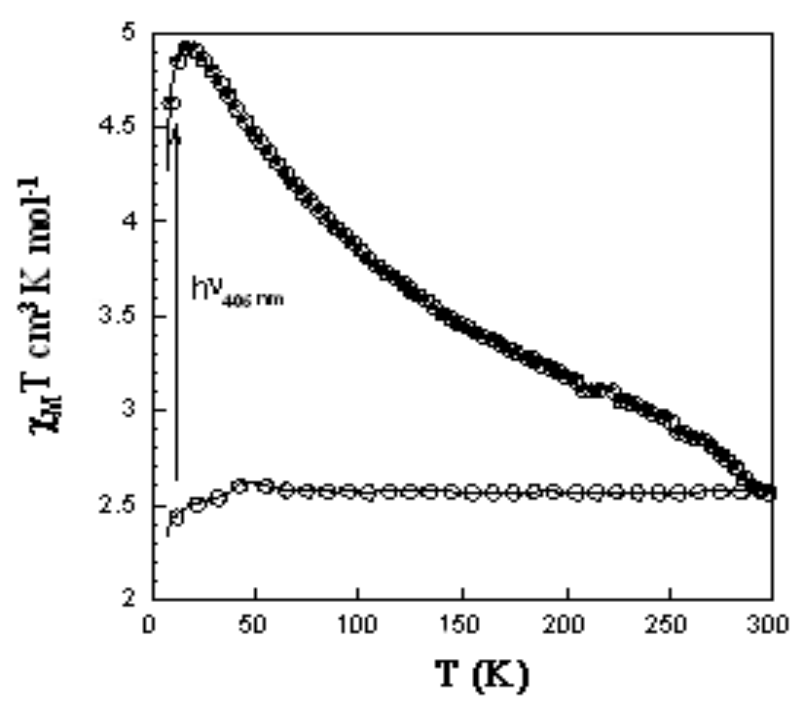

FIG. 1: Temperature dependence of $\chi_{M} T$, (०) before irradiation, (•) after irradiation.

six independent spin- $1 / 2$ objects per formula unit with $\chi_{M} T$ value of $2.48 \mathrm{~cm}^{3} \mathrm{~K} \mathrm{~mol}^{-1}$ at $20 \mathrm{~K}$. On irradiation with blue light $(406-415 \mathrm{~nm})$ at $10 \mathrm{~K}$, the $\chi_{M} T$ value increases to $4.8 \mathrm{~cm}^{3} \mathrm{~K} \mathrm{~mol}^{-1}$. On turning off the light and warming the system, the $\chi_{M} T$ value peaks at $5 \mathrm{~cm}^{3} \mathrm{~K}$ $\mathrm{mol}^{-1}$ and decreases thereafter gradually to a room temperature value of $2.6 \mathrm{~cm}^{3} \mathrm{~K} \mathrm{~mol}^{-1}$. The experimental data after irradiation is in agreement with $75 \%$ of the molecules in $\mathrm{S}=3$ state with the remaining in the state corresponding to six isolated $\mathrm{S}=1 / 2$ spins per molecule. Fig. 1] shows the $\chi_{M} T$ vs $T$ plot of the system before and after irradiation.

In this paper, we develop a microscopic quantum many-body model to understand the photomagnetic behaviour of this system. In the next section, we describe our model and the computational scheme in detail. In the third section we present the results from our model. We show that the eigenvalue spectrum of the model can be classified according to the occupancy of the various orbitals. The low-energy eigenvalue spectrum corresponds to one doubly occupied and one empty $M o$ orbital and singly occupied $C u$ orbitals with all the spin states in this sector being degenerate. In each of these spin sectors, we have several states to which approximately $3 \mathrm{eV}$ optical excitation has nonzero transition dipole moment. For a reasonable set of parameters the transition dipoles to the $\mathrm{S}=3$ excited states are the largest. We also find that the $\mathrm{S}=3$ density of states (DoS) is an order of magnitude smaller than the DoS in other spin sectors. This would lead to long life-time for the $\mathrm{S}=3$ optical excitations resulting in the system behaving like a $\mathrm{S}=3$ molecular complex after irradiation.

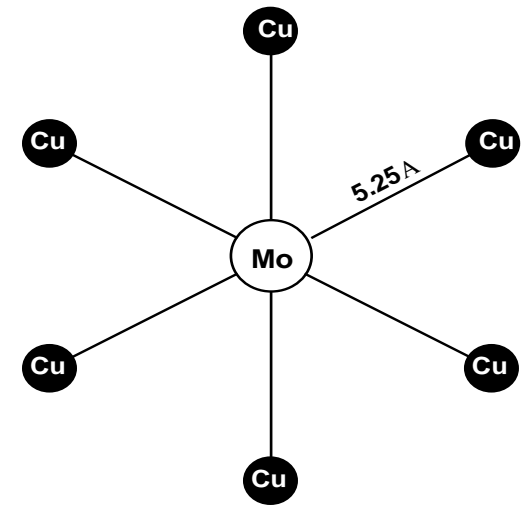

FIG. 2: Topology of the $\mathrm{MoCu}_{6}$ system with $M o$ ion at the center and $C u$ ions at the vertices of a regular hexagon. All the $M o-C u$ distances are 5.25 Åfrom $M o$.

\section{MICROSCOPIC MODEL}

The topology of the arrangement of the transition metal ions in the complex is hexagonal with the $M o$ ion at the center of the hexagon and the $\mathrm{Cu}$ ions at the vertices (Fig. 2). The molecular geometry is such that there is no electron transfer pathway which directly connects the copper ions, hence all exchange interactions between the $\mathrm{Cu}$ ions occur through the central $\mathrm{Mo}$ ion. Lowsymmetry of the complex around the $M o$ ion implies that the degeneracy of the $4 d$ orbitals on the $M o$ ion is lifted. This allows one to model photomagnetism in this system by considering only two low-lying $4 d$ orbitals on $M o$ and the partly occupied $3 d$ orbital on each $C u$. If we restrict the number of $M o$ orbitals to just one, no significant exchange interaction between the paramagnetic sites is possible since intra-atomic direct exchange interaction would be absent. Each $C u(I I)$ ion contributes one electron to the system while the $M o(I V)$ ion contributes two electrons. Thus, we would be dealing with an open shell system of eight electrons in eight orbitals.

\section{A. Model parameters}

The essential parameters of the model are the effective transfer parameter (the hopping integral) between the $C u$ orbital and each of the two orbitals on $M o$. It is assumed that the hopping integral, $t$, between a $C u$ orbital and any of the two active $M o$ orbitals is the same. The parameter $\Delta$ defines the orbital energy of the lower energy orbital on the $M o$ site, with reference to the orbital energy of the $C u d$-orbital taken to be zero. The energy splitting between the two orbitals on the $M o$ site is given by the parameter $\delta$. The quantities $t, \Delta$ and $\delta$ are all parameters of the non-interacting part of the Hamiltonian of the system.

The two-electron integrals, which are important for 
magnetism and should appear in the interacting part of the model Hamiltonian are the intra-orbital, the on-site inter-orbital and inter-site inter-orbital electron repulsion integrals. The first of these is given by,

$$
U^{i}=[i i \mid i i]=\iint \phi_{i}^{*}(1) \phi_{i}(1) \frac{1}{r_{12}} \phi_{i}^{*}(2) \phi_{i}(2) d^{3} r_{1} d^{3} r_{2}
$$

where $U^{i}$ is the familiar Hubbard parameter. We have assumed that the two Mo orbitals have the same Hubbard parameter. In cases where a given site has more than one orbital, we need to include inter-orbital Coulomb and exchange electron repulsion integrals in the model. There are three kinds of inter-orbital repulsion integrals, with two orbitals. These are denoted as $U^{i i^{\prime}}, X^{i i^{\prime}}$ and $W^{i i^{\prime}}$ and are given by,

$$
\begin{aligned}
U^{i i^{\prime}}=\left[i i \mid i^{\prime} i^{\prime}\right] & =\iint \phi_{i}^{*}(1) \phi_{i}(1) \frac{1}{r_{12}} \phi_{i^{\prime}}^{*}(2) \phi_{i^{\prime}}(2) d^{3} r_{1} d^{3} r_{2} \\
X^{i i^{\prime}} & =\left[i i^{\prime} \mid i i^{\prime}\right]=\iint \phi_{i}^{*}(1) \phi_{i^{\prime}}(1) \frac{1}{r_{12}} \phi_{i}^{*}(2) \phi_{i^{\prime}}(2) d^{3} r_{1} d^{3} r_{2} \\
W^{i i^{\prime}} & =\left[i i \mid i i^{\prime}\right]=\iint \phi_{i}^{*}(1) \phi_{i}(1) \frac{1}{r_{12}} \phi_{i}^{*}(2) \phi_{i^{\prime}}(2) d^{3} r_{1} d^{3} r_{2}(2)
\end{aligned}
$$

where we have used the charge cloud notation of chemists' to define the two electron integrals both here and elsewhere in the paper [9]. The single occupancy of the orbitals $\phi_{i}$ or $\phi_{i^{\prime}}$, is favoured over double occupancy of either, when the inter-orbital repulsion $U^{i i^{\prime}}$ is smaller than the Hubbard parameter $U^{i}$ or $U^{i^{\prime}}$ for either of the orbitals $\phi_{i}$ or $\phi_{i^{\prime}}$. The X integral is the exchange integral which favours parallel alignment of spins in the orbitals $\phi_{i}$ and $\phi_{i^{\prime}}$ when the orbitals are singly occupied, leading to the familiar Hund's rule. The $W^{i i^{\prime}}$ term does not play a significant role in magnetism but is included in the model for the sake of consistency since it is larger than the exchange integral. In our model, these terms arise only in the case of the $M o$ atom as it contributes two active orbitals to the model, say 1 and 2, thus we have $U^{12}=[11 \mid 22], W=[11 \mid 12]$ and $X=[12 \mid 12]$ 10]. We also include the extended range electron correlation corresponding to repulsion between charge densities located on different sites, within the zero differential overlap approximation, via Ohno interpolation [11]. The model Hamiltonian can now be written as

$$
\begin{aligned}
\hat{H}= & t \sum_{i=3}^{8}\left(\hat{E}_{1, i}+\hat{E}_{2, i}+H . c .\right)+\Delta \hat{n}_{1}+(\Delta-\delta) \hat{n}_{2} \\
& +\sum_{i=1}^{8} U^{i} \hat{n}_{i}\left(\hat{n}_{i}-1\right) / 2+U^{12} \hat{n}_{1} \hat{n}_{2} \\
& +\sum_{i=3}^{8}\left(V_{1 i} \hat{n}_{1} \hat{n}_{i}+V_{2 i} \hat{n}_{2} \hat{n}_{i}\right) \\
& +\frac{W}{2}\left[\left(\hat{n}_{1}+\hat{n}_{2}\right)\left(\hat{E}_{12}+\hat{E}_{21}\right)+\left(\hat{E}_{12}+\hat{E}_{21}\right)\left(\hat{n}_{1}+\hat{n}_{2}\right)\right. \\
& \left.-2\left(\hat{E}_{12}+\hat{E}_{21}\right)\right]
\end{aligned}
$$

$$
\begin{aligned}
& +\frac{X}{2}\left(\hat{E}_{12} \hat{E}_{12}+\hat{E}_{12} \hat{E}_{21}+H . c+\hat{E}_{21} \hat{E}_{21}\right. \\
& \left.-\hat{n}_{1}-\hat{n}_{2}\right)
\end{aligned}
$$

Here, H.c. stands for Hermitian conjugate; our numbering scheme for the orbitals is such that orbitals 1 and 2 are on $M o$ site and orbitals 3 through 8 are each located on the copper sites and

$$
\hat{E}_{i, j}=\sum_{\sigma} \hat{a}_{i, \sigma}^{\dagger} \hat{a}_{j, \sigma} ; \hat{n}_{i}=\sum_{\sigma} \hat{a}_{i, \sigma}^{\dagger} \hat{a}_{i, \sigma}
$$

The inter-site interaction $V_{i j}$ is parametrized as

$$
V_{i j}=14.397\left[\left(\frac{28.794}{U^{i}+U^{j}}\right)^{2}+r_{i j}^{2}\right]^{-\frac{1}{2}}
$$

with the distance $r_{i j}$ is in $\AA$ and energies are in $\mathrm{eV}$. The geometry of the cluster $\mathrm{MoCu}_{6}$ is taken to be a regular hexagon with a $M o-C u$ distance of $5.25 \AA$. The topology of the complex used in modeling the system is shown schematically in Fig. 2

\section{B. Computational details}

The model Hamiltonian spans a finite dimensional Hilbert space corresponding to eight electrons in eight orbitals. Since the Hamiltonian conserves total spin, the Hilbert space can be further subdivided into spaces of total spin $0,1,2,3$ and 4 whose dimensions are 1764, 2352, 720, 63 and 1 respectively. The basis states with a given total spin can be generated using a valence bond approach of explicit spin pairings [12]. For example, if singly occupied sites $i$ and $j$ are spin paired, then a line is drawn between sites $i$ and $j$ in the VB diagram to indicate the spin pairing, $\left(\alpha_{i} \beta_{j}-\beta_{i} \alpha_{j}\right) / \sqrt{2}, i<j$. We say that the line begins at site $i$ and ends at site $j$. If the spins at sites $i_{1}, i_{2} \cdots i_{l}$ are not paired, we pass an arrow through these sites in the VB diagram and this denotes the state $\alpha_{i_{1}} \alpha_{i_{2}} \cdots \alpha_{i_{l}}$. Because the Hamiltonian conserves $S_{\text {total }}^{z}$ besides $S^{2}$, it is sufficient to work in the subspace $M_{S}=S$. The complete and linearly independent set of VB states in each spin space can be obtained by taking recourse to modified Rumer-Pauling rules [13]. Some typical VB diagrams are shown in Fig. 3 Each orbital in the VB picture has one of four possibilities; the orbital is (i) empty, (ii) doubly occupied, (iii) a line begins at the orbital or (iv) a line ends at the orbital. It is possible to associate these four possibilities of an orbital in a VB diagram with the four states of two bits and stored as sixteen bit integers in an ascending order. In any given spin space, the effect of the term $\hat{E}_{i j}$ in the Hamiltonian on a basis state is to alter the orbital occupancy of orbitals $i$ and $j$ (subject to Pauli principle) and pair the spins in the orbitals that were involved to yield a new VB digram with a fixed amplitude. If the new 
(a)

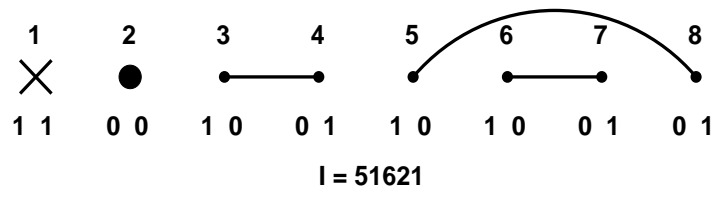

(b)

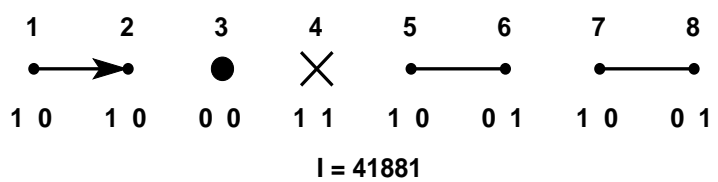

(c)

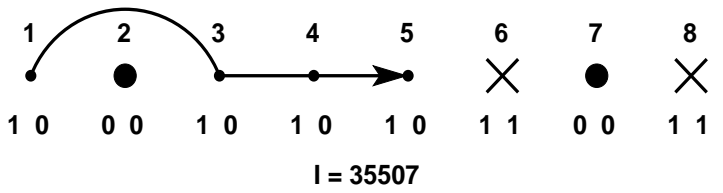

(d)

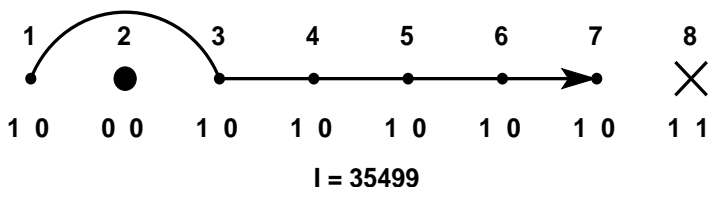

FIG. 3: Representative VB diagrams in (a) $\mathrm{S}=0$, (b) $\mathrm{S}=1$, (c) $\mathrm{S}=2$ and (d) $\mathrm{S}=3$ subspaces. Dots $(\bullet)$ represent empty sites and crosses $(x)$ represent doubly occupied sites. In each VB diagram, numbers 1 through 8 are the site indices and the values at the bottom against each site gives the bit state of the bits representing the site in the integer I. The bit-pattern and the value of the integer I, representing the VB diagram are also shown.

VB diagram violates Rumer-Pauling rules, it is trivially possible to express it as a linear combination of the basis VB states.

Using the above procedure, the Hamiltonian matrix can be set-up in the chosen total spin sector. The resulting Hamiltonian matrix is sparse (since the number of terms in the Hamiltonian is far smaller than the dimension of the VB complete space) and nonsymmetric matrix and can be partially diagonalized to obtain a few of the low-lying eigenstates, using Rettrups modification of the Davidson algorithm [14, 15]. In some cases, full diagonalization of the matrices is resorted to obtain the complete eigenvalue spectrum. In our case, since the dimensionalities of the different subspaces are fairly small, we have resorted to complete diagonalization of the Hamiltonian matrices in each spin sector.

In the present problem, the quantities of interest are the optical gaps in each spin sector and the transition dipoles for these transitions. To understand the nature of the excited states, it is also important to know the spin and charge density distributions in these states. Computing many of these quantities is simpler in the constant $M_{S}$ basis. Hence, we transform eigenstates in the va- lence bond basis to the constant $M_{S}$ basis and carry out all these calculations.

\section{RESULTS AND DISCUSSION}

We have extensively explored the properties of the model over a wide range of parameters, specifically, we have studied the model at discrete values of the parameters in the following ranges: $4 \mathrm{eV} \leq U^{C u} \leq 6 \mathrm{eV}, 0.5 \mathrm{eV}$ $\leq U^{C u}-U^{M o} \leq 2.0 \mathrm{eV},-0.2 \mathrm{eV} \leq \Delta \leq-0.5 \mathrm{eV}, 0.0 \mathrm{eV}$ $\leq \delta \leq 0.3 \mathrm{eV}, 0.5 \mathrm{eV} \leq U^{M o}-U^{M o}-M o$ $\leq W \leq 1.5 \mathrm{eV}$ with $X \leq W / 2$. However, we report our results for the model parameters values of $U^{C u}=6.0 \mathrm{eV}$, $U^{M o}=4.0 \mathrm{eV}, \Delta=-0.4 \mathrm{eV}, \delta=0.0 \mathrm{eV}, U^{M o-M o}=3.5 \mathrm{eV}$, $W=0.75 \mathrm{eV}$ and $X=0.3 \mathrm{eV}$ since it gives a reasonable value for the optical gap of the system. Our assumption of $U^{C u}$ being higher than $U^{M o}$ is justified since $3 d$ orbitals are more compact than the $4 d$ orbitals. The interelectron repulsion integral involving the $\mathrm{d}$ orbitals on $M o,\left(U^{M o-M o}\right)$ is slightly less than the intra-orbital repulsions. We expect the integral $W$ to be larger than $X$, and both are expected to be significantly smaller than the $U$ type repulsion integrals that involve repulsion between two electron densities, each one located in a single orbital. The orbital energy of the Mo $4 d$ orbitals could be slightly smaller than the $C u 3 d$ orbital energy since $M o$ has a higher atomic number. Therefore, we have assumed $\Delta$ to be small and negative. These values of the interaction parameter are reasonable as found from theoretical studies on transition metal oxides in the context of high- $\mathrm{T}_{c}$ superconductors [16]. For these model parameters values, transfer integrals in the range $6 \times 10^{-4}$ $\mathrm{eV} \leq t \leq 0.01 \mathrm{eV}$ fit the experimental $\chi T$ vs $T$ data. This is because, the various spin states with all Copper ions in +2 oxidation state and Molybdenum ion in +4 oxidation state are degenerate to within about $t^{2} / U$, where $\mathrm{U}$ is an average Hubbard parameter $(\sim 5 \mathrm{eV})$. The near degeneracy of these "covalent" spin states ensures that the behaviour for $T>t^{2} / U$ corresponds to that of six free spins on the $C u(I I)$ ions. We have fixed the value of $t$ at $0.01 \mathrm{eV}$ to ensure that there is a significant transition dipole between the low-lying spin state and its optically coupled state.

\section{A. Low-energy spectrum of the model}

To understand the eigenvalue spectrum of the model, it is instructive to analyze the $t=0$ behaviour. In this case, the eigenstates of the model can be completely classified based on the site occupancies. The only off-diagonal contribution that mixes the states with different occupancies arises from the $W$ and the $X$ terms involving the $M o$ orbitals. These terms, however, conserve the total number of electrons on $M o$. When we have the $4 d^{2}$ configuration 


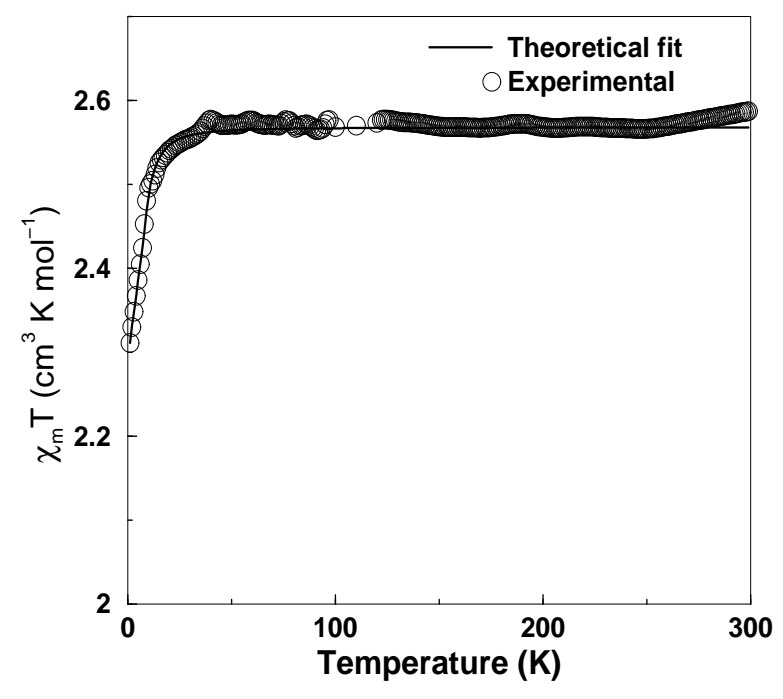

(a)

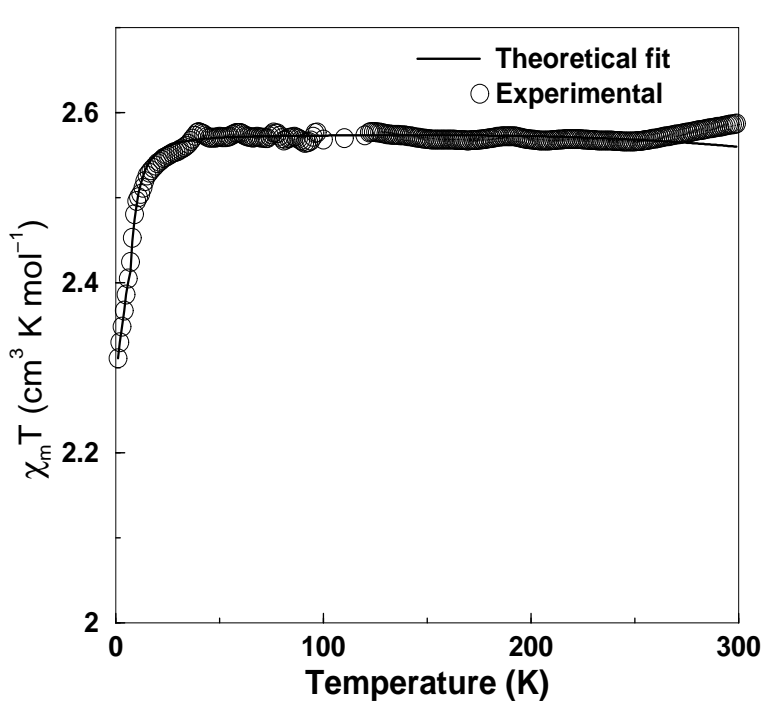

(b)

FIG. 4: Theoretical fit of temperature dependence of $\chi_{M} T$ for (a) $t=0.01 \mathrm{eV}$ and (b) $t=0.00065 \mathrm{eV}$. Error in the fit $R$ calculated from $R=\sum_{i}\left(\chi_{\text {expt }}\left(T_{i}\right)-\chi_{\text {calc }}\left(T_{i}\right)\right)^{2} / \sum_{i} \chi_{\text {expt }}\left(T_{i}\right)^{2}$ for (a) $1 \times 10^{-5}$ and (b) $1.35 \times 10^{-5}$.

on $M o$ and all the copper ions in $3 d^{9}$ configuration, we have a total of 384 states. This corresponds to six ways of occupying two electrons in the two $4 d$ orbitals and sixty four spin configurations of the six spins on the six $C u(I I)$ ions. For the model employed by us, the lowest energy state corresponds to the lower of the orbitals on Mo being doubly occupied, for $U^{M o}<\left(U^{M o-M o}+\delta\right)$, ignoring the off-diagonal contributions from the $W$ and $X$ terms. The effect of the off-diagonal $W$ and $X$ terms is (i) to favour double occupancy on the lower energy $M o$ orbital and (ii) to favour parallel spin alignment when these orbitals are singly occupied. Corresponding to this occupancy, five states with total spin $\mathrm{S}=0$, nine states with total spin $S=1$, five states with total $S=2$ and one state with total spin $\mathrm{S}=3$ are all degenerate. There is only one $\mathrm{S}=4$ state which is $0.671 \mathrm{eV}$ above the ground state and corresponds to single occupancy of each orbital with all spins aligned in parallel. These states span all the 64 spin configurations corresponding to six spin- $1 / 2$ objects; since all these states are degenerate to within a few degree $\mathrm{K}$, the paramagnetic behaviour observed in the system before irradiation is reproduced for small values of $t$. The theoretical fit to experimental data for two different values of the transfer integral is shown in Fig. 4

The next set of states corresponds to single occupancy of each of the $M o$ orbitals. If the spins of the electrons in these orbitals are parallel, the states will have a lower energy than if they are antiparallel. There are in all 256 such states of which 128 states have parallel spin alignment and another 128 states have antiparallel spin alignment. The remaining sixty four of the 384 states have a double occupancy in the higher energy $M o$ orbital.
These 384 states form the low energy spectrum when $U^{C u}$ is significantly larger than $\left(U^{M o}-\Delta\right)$ so that the intersite interactions do not alter the picture. The most important point to note is that since the low-energy states all have identical charge distribution, the transition dipole matrix elements between these states are zero and this result holds even when $t \neq 0$.

\section{B. Charge-Transfer Excited States}

The higher energy excited states of the system involve charge-transfer $(\mathrm{CT})$ from the $M o(I V)$ ion to $C u(I I)$ resulting in a state with a large weight for the $M o(V)-C u(I)$ configurations. These states are found at about $3 \mathrm{eV}$ above the ground state. These excitations have nonzero transition dipole to the ground state. One of the most important features of this excitation is that, in the excited state there is considerable weight for the basis state with singly occupied Mo site. The singly occupied $M o(V)$ site favours virtual electron transfer from the $C u(I I)$ sites, of electrons which have the same spin orientation as the unpaired $M o(V)$ electron, because of the Hund's rule. The virtual transfer of an electron from $C u(I I)$ with its spin orientation same as that already present on $M o(V)$ is favoured as long as (i) the $d$-orbitals on $M o$ are nearly degenerate and (ii) the inter-orbital $d-d$ repulsion, on $M o$ is weaker than the intra-orbital repulsion $\left(U^{M o-M o}<U^{M o}\right)$. This feature contributes to stabilizing the high-spin CT excitation relative to the low-spin excitations. In Fig 5 we show the possible pathways for superexchange in this state. The factor which favours stabilization of the low-spin state 


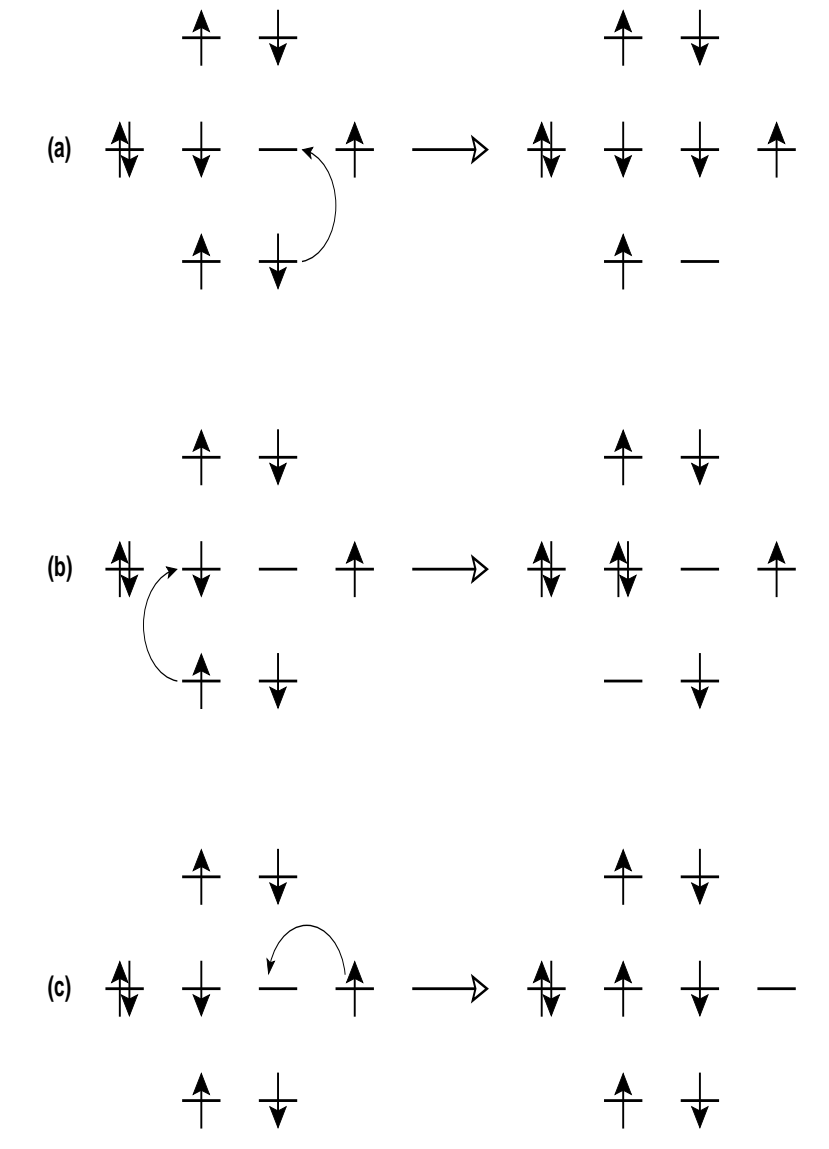

FIG. 5: The virtual excited state resulting from the electron transfer shown in (a) has a lower energy than those shown in (b) and (c), because of the Hund's rule. The superexchange process in (a) favours $\mathrm{S}=3$ state, and in (b) an $\mathrm{S}=2$ state. The process (b) is preferred over (a) if the splitting of the orbitals on the $M o$ site as well as $U^{M o-M o}$ are large. In all the figures, the two orbitals in the center are the $M o$ orbitals and the six peripheral orbitals are the $C u$ orbitals.

is the large number of pathways that exist for the delocalization of electrons in the low-spin state, compared to the high-spin states. In our case, there are 1764 singlets and 2352 triplets while there are only $63 \mathrm{~S}=3$ states. The splitting of the states with different spins in the CT state is thus a competition between direct exchange which stabilizes the high-spin state and the kinetic exchange which favours the low-spin state due to the availability of a larger phase space for electron delocalization. This implies that the lowest-energy $\mathrm{CT}$ excited state could have any spin, $S$, in the range $0 \leq \mathrm{S} \leq 3$.

If the splitting of the Mod orbitals is large or $U^{M o-M o}$ is large, then the intermediate virtual state with a doubly occupied $d$ orbital on $M o$ is favoured, resulting in an antiferromagnetic interaction between the $C u(I I)$ ion and the $M o$ spin (Fig 5 . This factor would contribute to a lowering of the $\mathrm{S}=2 \mathrm{CT}$ excited state relative to $\mathrm{CT}$ excited states with spin $\mathrm{S} \neq 2$. However, the final outcome depends on both the direct exchange and the kinetic ex-
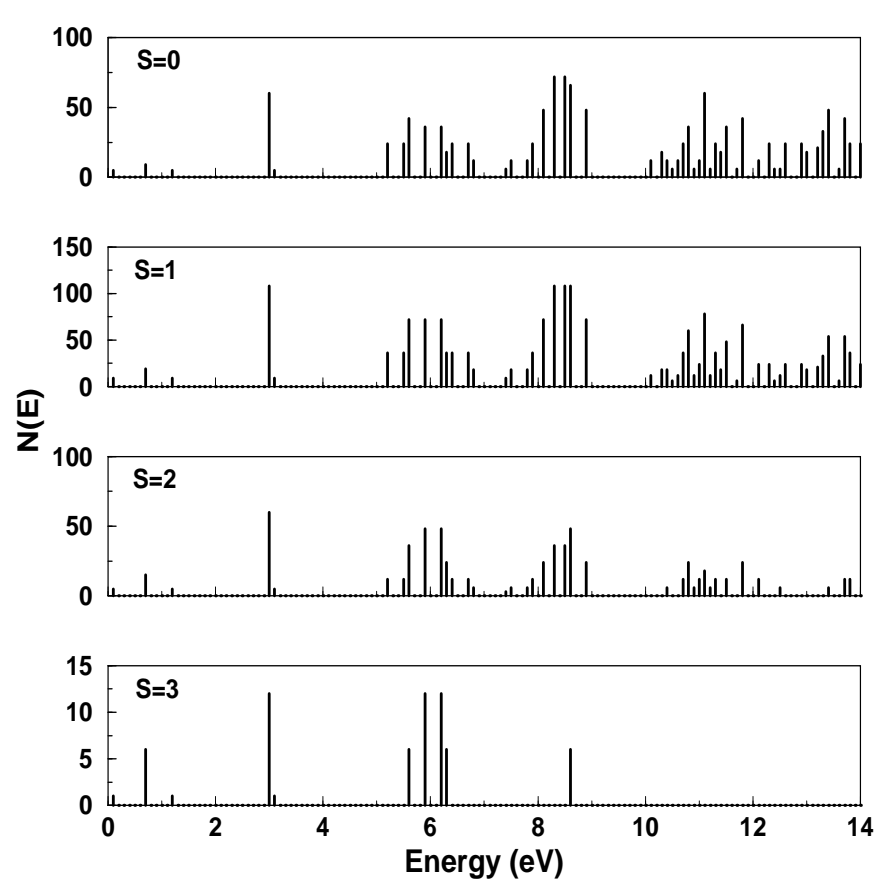

FIG. 6: Density of states versus energy in $\mathrm{S}=0,1,2$ and 3 subspaces. There is only one state in $\mathrm{S}=4$ sector at $0.6709 \mathrm{eV}$ from the ground state (not shown in the figure).

change contributions. Thus, it is again difficult to predict which of the spin states is a low-energy CT excited state. We see all the above scenarios in our calculations, when we widely vary the model parameters.

The above picture is slightly altered by the long-range interactions in the model. These interactions depend upon the occupancies of the Mo site as well as all the $\mathrm{Cu}$ sites and are significant since the molecular complex is insulating and screening of these interactions is not significant. Together with a nonzero transfer integral, the long-range interactions complicate the picture while retaining the general physical features outlined above.

For the model parameter for which we are reporting the results, we show the histogram giving the number of states as a function of energy in Fig. 6] for the full spectrum of the model in each total spin sector. We find CT excitations at about $2.993 \mathrm{eV}$ in all the spin sectors. The energies and magnitude of the transition dipole vectors from the ground state are given in Table \. We also present in Table $\llbracket$ the degeneracy of states as well as the charge and spin density on the $M o$ site. We note that in all the states located at this energy, independent of the total spin, the charge density on the $M o$ site is one. What is interesting is that while the energies of these states as well as the charge density on the Mo site are all the same, the transition dipole moment is largest for the $\mathrm{S}=3$ states. This fact, coupled with the high degeneracy of the state implies that the $\mathrm{S}=3$ states have the largest absorption to the CT excited state. 
TABLE I: Energy gaps (in eV) to the charge-transfer excited states from the lowest state in different spin sectors and the magnitude of the corresponding transition dipole moments in $10^{-3}$ Debye. Also presented in the table are the total spin and charge densities on the Molybdenum site in the excited state.

\begin{tabular}{|c|c|c|c|c|c|}
\hline Spin sector & Energy gap (eV) & Degeneracy & $\begin{array}{c}\text { Transition dipole } \\
\left(\mathrm{x} 10^{-3} \text { Debye }\right)\end{array}$ & $\left\langle S_{z}^{M o}\right\rangle$ & $\left\langle n_{M o}\right\rangle$ \\
\hline $\mathrm{S}=0$ & 2.9921 & 6 & 7.0 & 0.0000 & 1.0000 \\
& 2.9923 & 4 & 4.1 & 0.0000 & 1.0000 \\
\hline \multirow{3}{*}{$\mathrm{S}=1$} & 2.9920 & 4 & 6.2 & -0.2498 & 1.0000 \\
& 2.9923 & 8 & 5.3 & 0.4986 & 1.0000 \\
& 2.9924 & 2 & 3.4 & 0.5000 & 1.0000 \\
\hline \multirow{3}{*}{$\mathrm{S}=2$} & 2.9921 & 2 & 8.3 & -0.1596 & 1.0000 \\
& 2.9923 & 2 & 12.1 & 0.5000 & 1.0000 \\
& 2.9924 & 4 & 7.8 & 0.5000 & 1.0000 \\
\hline $\mathrm{S}=3$ & 2.9925 & 5 & 16.0 & 0.5000 & 1.0000 \\
\hline
\end{tabular}

An important difference between the $\mathrm{S}=3$ eigenvalue spectrum and eigenvalue spectra of other spin states is that the DoS in the former is very dispersed and the states are far apart in energy, when not degenerate. This has implications for the life-time of the excited states. In the $\mathrm{S}=0,1$ and 2 spaces, there are an order of magnitude more states near the CT excitation gap of 2.993 eV compared with the $\mathrm{S}=3$ states at this energy. Thus, other spin states have pathways for internal conversion leading to rapid de-excitation of the CT state. This is less likely in the $\mathrm{S}=3$ state. For this reason, the $\mathrm{S}=3$ excited state has a longer life-time and can be observed in the photomagnetism experiments. One feature that is however missed in our studies is the computation of the equilibrium geometry in the CT excited state specifically around the $C u(I)$ site that may play a crucial role in increasing the life-time of the state. This is rather hard to incorporate in our model wherein the ligand atoms are completely neglected. It is possible that the CT excited state with $\mathrm{S}=3$ is more distorted (relative to the ground state) than similar states with different total spin. This could further enhance the life-time of the $\mathrm{S}=3 \mathrm{CT}$ excitation.

For the sake of completeness, it is worth mentioning that there are also different kinds of charge transfer excited states at much higher energies corresponding to electron transfer from the $C u(I I)$ site to the $M o(I V)$ site, charge transfer between $C u(I I)$ sites and so forth for which the transition dipole moments are nonzero. However, these states occur at a much higher energy and are not excited by laser light at $3 \mathrm{eV}$.

The model developed in this paper is specific to the isolated molecular complex we have studied. However, this model also throws light on the mechanism leading to the observed ferromagnetism in the PIPT systems. While the degeneracy of the different optically excited spin states is not significantly affected by the exchange mechanism operative in the optically excited state, the long life-time of the high-spin state could be responsible for the observed ferromagnetism in extended systems.

\section{CONCLUSIONS}

In this paper, we have introduced a model for the photomagnetism in the heptanuclear complex, $\left[M o(I V)(C N)_{2}(C N-C u L)_{6}\right]^{8+}$ perchlorate which involves the six partly filled $3 d$ orbital on the $C u(I I)$ sites and two lowest energy $4 d$ orbitals on the $M o(I V)$ site. Besides the site energies of the orbitals and the transfer integrals between the $C u$ and $M o$ orbitals, we introduce on-site and extended range interactions between the various sites. On the $M o$ site, we consider all the three types of two electron integrals involving the $M o$ orbitals. Using this model, we compute the full eigenvalue spectrum, the transition dipoles with the ground state, spin and charge densities for all the states in each of the spin sectors by exactly solving the model in a spin conserving valence bond basis. Our studies show that the ground state is sixty four fold degenerate with an unpaired electron on each $C u$ site. This leads to the observed paramagnetic susceptibility prior to irradiation, of six non-interacting spin-1/2 objects per formula unit. The excited states to which there is nonzero transition dipole in each spin sector are located at about $2.993 \mathrm{eV}$ (for the values of the parameter we have chosen) in each of the spin sectors. The number of states at this energy as well as the transition dipole moment for the transition are highest for the $\mathrm{S}=3$ states. There also exist very few states in the $\mathrm{S}=3$ sector below this energy, thereby introducing a bottleneck for its internal conversion for non-radiative decay to the ground state. There may also occur changes in the geometry of the complex in the excited state which renders a low Frank-Condon factor for radiative decay. Thus, the observed post radiation magnetic behaviour of the complex with $75 \%$ of the molecules in the $\mathrm{S}=3$ state can be rationalized by our model. 


\section{ACKNOWLEDGMENT}

The authors thank DST for the support received under a joint Indo-French Laboratory for Solid State Chemistry (IFLaSC) and Indo-French Centre for Promotion of Advanced Research (IFCPAR) for generous support under Project 3108-3 on Design, Synthesis and Modeling Molecular Magnets. The authors also thank the French ministry of research (ACI young researcher No JC4123) and the European Community (Network of Excellence, MAGMANet) for the financial support.

[1] Y. Ogawa, S. Koshihara, K. Koshino, T. Ogawa, C. Urano and H. Takagi, Phys. Rev. Lett. 84(14), 3181 (2000).

[2] G. Rombaut, M. Verelst, S. Golhen, L. Ouahab, C. Mathoniére, O. Kahn, Inorg. Chem., 40, 1151 (2001); S. Ohkoshi, N. Machida, Z. J. Zhong, K. Hashimoto, Synth. Met., 122, 523 (2001); S. Ohkoshi, N. Machida, Y. Abe, Z. J. Zhong, K. Hashimoto, Chem. Lett., 30, 312 (2001).

[3] Osamu Sato, Acc. Chem. Res., 36, 692-700 2003.
[4] Y.Tanabe and S.Sugano, J. Phys. Soc. Japan, 9, 753 and 766 (1954).

[5] Philipp Gütlich, Yann Garcia and Theo Woike, Coord. Chem. Rev. 219-221, 839 (2001);

[6] O. Sato, T. Iyoda, A. Fujishima and K. Hashimoto, Science 272, 704 (1996).

[7] T. Yokoyama, M. Kiguchi, T. Ohta, O. Sato, Y. Einaga, K. Hashimoto, Phys. Rev. B, 60, 9340-9346 (1999).

[8] Juan Manuel Herrera, Valérie Marvaud, Michel Verdaguer, Jérôme Marrot, Marguerite Kalisz and Corine Mathoniere, Angew. Chem. Int. Ed. 43, 5468 (2004).

[9] Attila Szabo, Neil. S. Ostlund, Modern Quantum Chemistry - Introduction to Advanced Electronic Structure Theory, Dover Publications Inc., New York (1996).

[10] R. Pariser and R. G. Parr, J. Chem. Phys. 21, 466 (1953); J. A. Pople, Trans. Faraday Soc. 49, 1375 (1953);

[11] K. Ohno, Theor. Chim. Acta 2, 219 (1964); G. Klopmann, J. Am. Chem. Soc. 86, 4450(1964).

[12] S. Ramasesha and Z. G. Soos, In: David L. Cooper (Ed.), Valence Bond Theory, Elsevier Science, Amsterdam, Chapter 20, 635 (2002).

[13] G. Rumer, Nachr. Chem. Tech. Goettingen, 377(1932).

[14] E. R. Davidson, J. Comp. Phys. 17, 87 (1975).

[15] S. Rettrup, J. Comp. Phys. 45, 100 (1982).

[16] S. R. Barman, D. D. Sarma, Phys. Rev. B. 49(19), 13979 (1994). 